\title{
A review on the Idylla platform: towards the assessment of actionable genomic alterations in one day
}

\author{
Arnaud Uguen, ${ }^{1,2}$ Giancarlo Troncone ${ }^{3}$
}

'Department of Pathology, CHRU Brest, Brest, France ${ }^{2}$ Inserm U1053 BaRITOn, Bordeaux, France

${ }^{3}$ Department of Public Health, Anatomic Pathology Unit, University of Napoli Federico II, Napoli, Italy

\section{Correspondence to} Dr Arnaud Uguen, Department of Pathology, University Hospita Morvan, Brest 29609, France; arnaud.uguen@chu-brest.fr

Received 30 March 2018 Revised 18 May 2018 Accepted 22 May 2018 Published Online First 14 June 2018

Check for updates

To cite: Uguen $A$, Troncone G. J Clin Pathol 2018:71:757-762

\section{INTRODUCTION}

Cancer still represents a major cause of death in the world. In the last decades, a better understanding of the molecular bases of cancer initiation, progression and antitumour immunity response escape has led to the development of new therapeutic strategies. Indeed, targeted treatments against oncogenic proteins and immune-checkpoint inhibitors have considerably improved quality of life and treatment outcomes of patients with cancer. Thus, assessment of predictive biomarker is crucial. According to the US Food and Drug Administration, a companion diagnostic assay, which provides essential information for the safe and effective use of a corresponding drug, is a unique medical device. Conversely, in the European Union the same biomarker can be analysed by using different diagnostic platforms, with different advantages and drawbacks. Nevertheless, any commercially available diagnostic assay needs to achieve validation, for approval by the European Community for in vitro diagnostic use (CE-IVD).

Although the next-generation sequencing (NGS) of even whole exomes or genomes is today feasible in expert centres, currently only limited pieces of genomic information are clinically relevant for the therapeutic management of patients with advanced cancers. Indeed, besides clinical trials and experimental protocols, the list of predictive actionable genomic biomarkers is quite short to date. Relevant examples include in colorectal cancer mutations of KRAS and NRAS; in lung cancer, mutations of EGFR and rearrangements of $A L K$ and ROS1; and in melanoma BRAF and NRAS mutations. ${ }^{1-8}$ Reflecting the requirements of expensive equipments, experience and skilled personnel, these mutational assays are often centralised in reference laboratories. The longer interval of time between test prescription and result delivery may delay the treatment of patients with advanced cancers, some of them suffering from acute deterioration and needing rapid therapeutic decisions. The Idylla system (Biocartis, Mechelen, Belgium) can be easily implemented in pathology laboratories to diagnose quickly and simply oncogenic mutations. ${ }^{9-26}$ This methodology consists of a cartridge-based fully automated medical device able to perform molecular analyses in $<1$ day even in laboratory without experience in molecular analyses. Briefly, formalin-fixed and paraffin embedded (FFPE) tissue sections or even cytological material are placed in a single-use cartridge, itself placed in the Idylla platform and the whole real-time quantitative PCR procedure is performed automatically inside the cartridge, from the DNA extraction to the assay interpretation. In the last few years, a large body of data has accumulated on the various facets of this innovative technology. Hence, it seems the time is now ripe to review the technical and clinical aspects of the Idylla system as an alternative or a complementary molecular diagnostic tool in oncology applications discussing its advantages and limitations in the management of patients with advanced cancers.

\section{METHODS}

The MEDLINE and Google Scholar databases were searched to retrieve studies addressing the Idylla system performance compared with other diagnostic methods. Only original papers were taken into account, excluding congress abstracts. Data analysed included the number and the types of samples, the specific Idylla cartridges employed and the non-Idylla reference method; special care was also taken to record any case showing discordance, focusing on the underlining reasons leading to Idylla and non-Idylla methods disagreements.

\section{RESULTS}

\section{Overview of the different studies}

As shown in table 1, 18 original studies evaluated the performances of the Idylla system compared with other methods. ${ }^{9-26}$ These latter widely differed among studies, and in some instances, besides a firstline reference method an additional third method was used to further analyse discrepant results. Overall, five studies were dedicated to colorectal cancer, four to lung cancer, four to melanoma, one to thyroid cancer, one to pancreatic cancer and three to different tumours including the aforementioned types as well as a few examples of other tumours. FFPE tumour samples were analysed in 15 studies, including one report analysing both tissue microarray core biopsies and matched whole tissue sections. In three additional studies, non formalin-fixed cytological material was used. BRAF was the only mutational test assessed in seven studies, whereas its association with NRAS or with NRAS and KRAS testing was evaluated in two and one studies, respectively. KRAS assay was studied as the sole diagnostic test in four studies and in association with EGFR testing in the other two studies. In one of these, a sequential strategy was based on patient smoking history as EGFR and KRAS were tested first in non-smokers and smokers, respectively. ${ }^{10}$ Two studies only analysed EGFR mutational testing. 
Table 1 Summary of the studies comparing Idylla with other diagnostic methods in cancer samples

\begin{tabular}{|c|c|c|c|c|c|c|c|c|c|}
\hline Reference & $\begin{array}{l}\text { Tumour } \\
\text { types }\end{array}$ & $\begin{array}{l}\text { Sample } \\
\text { types }\end{array}$ & Idylla cartridges & $\begin{array}{l}\text { Samples } \\
\text { analysed }\end{array}$ & $\begin{array}{l}\text { Idylla non- } \\
\text { contributive } \\
\text { results }\end{array}$ & $\begin{array}{l}\text { Concordant } \\
\text { results between } \\
\text { Idylla and } \\
\text { the reference } \\
\text { method }\end{array}$ & $\begin{array}{l}\text { Discrepant } \\
\text { results } \\
\text { between } \\
\text { Idylla and } \\
\text { the reference } \\
\text { method }\end{array}$ & $\begin{array}{l}\text { Idylla true } \\
\text { results } \\
\text { according } \\
\text { to a third } \\
\text { evaluation }\end{array}$ & $\begin{array}{l}\text { Idylla false } \\
\text { results } \\
\text { according } \\
\text { to a third } \\
\text { evaluation }\end{array}$ \\
\hline 9 & Melanoma & FFPE & Idylla BRAF Mutation Test & 148 & 7 & 126 & 14 & 8 & 1 \\
\hline 23 & Various & FFPE & Idylla BRAF Mutation Test & 216 & 0 & 210 & 6 & 2 & 0 \\
\hline 21 & Various & FFPE & Idylla BRAF Mutation Test & 436 & 15 & 406 & 13 & 6 & 1 \\
\hline 20 & Thyroid & FFPE & Idylla BRAF Mutation Test & 110 & 0 & 99 & 11 & 10 & 1 \\
\hline 16 & Melanoma & FFPE & Idylla BRAF Mutation Test & 37 & 0 & 36 & 1 & 1 & 0 \\
\hline 17 & Colorectal & FFPE & Idylla NRAS-BRAF Mutation Test & 242 & 0 & 241 & 1 & 1 & 0 \\
\hline 26 & Melanoma & FFPE & $\begin{array}{l}\text { Idylla NRAS-BRAF-EGFRS492R } \\
\text { Mutation Assay }\end{array}$ & 36 & 0 & 35 & 1 & 0 & 1 \\
\hline 11 & Colorectal & FFPE & Idylla KRAS Mutation Test & 252 & 7 & 174 & 20 & 13 & 7 \\
\hline 24 & Colorectal & FFPE & Idylla KRAS Mutation Test & 374 & 2 & 347 & 24 & 6 & 16 \\
\hline 12 & Pancreatic & Cyto. & Idylla KRAS Mutation Test & 21 & 3 & 18 & 0 & 0 & 0 \\
\hline 14 & Pancreatic & Cyto. & Idylla KRAS Mutation Test & 52 & 3 & 42 & 7 & 0 & 7 \\
\hline \multirow[t]{2}{*}{10} & \multirow[t]{2}{*}{ Lung } & \multirow{2}{*}{$\begin{array}{l}\text { FFPE and } \\
\text { fresh }\end{array}$} & Idylla EGFR Mutation Assay & 68 & 3 & 63 & 1 & 0 & 1 \\
\hline & & & Idylla KRAS Mutation Test & 73 & 3 & 63 & 7 & 0 & 7 \\
\hline \multirow[t]{2}{*}{19} & \multirow[t]{2}{*}{ Lung } & \multirow[t]{2}{*}{ FFPE } & Idylla EGFR Mutation Assay & 18 & 0 & 15 & 3 & 0 & 3 \\
\hline & & & Idylla KRAS Mutation Test & 18 & 0 & 18 & 0 & 0 & 0 \\
\hline 13 & Lung & Cyto. & Idylla EGFR Mutation Assay & 76 & 2 & 70 & 4 & 2 & 0 \\
\hline 15 & Lung & FFPE & Idylla EGFR Mutation Assay & 110 & 0 & 104 & 6 & 6 & 0 \\
\hline
\end{tabular}

Cyto, cytological samples; fresh, unfixed samples; FFPE, formalin fixed paraffin-embedded samples; Reference, reference of the study in the review.

Considering all 18 studies, a total of 2482 Idylla tests were performed in tumour samples obtained from 2343 patients. Idylla methodology did not yield contributive results in $1.9 \%$ $(48 / 2482)$ of the cases, which was a less frequent occurrence when considering the reference methods (3.1\%; 76/2482). Overall, in 2378 instances Idylla and reference method paired results were available for comparison, with a concordance rate of $94.8 \%(2255 / 2378)$. Discrepancies were obtained in 123 instances (5.2\%). Most (84.6\%) of these cases (104/123) were further resolved by a third method. The Idylla result was confirmed in 54.8\% (57/104), whereas discrepancies were still observed in $46.2 \%(47 / 104)$ of cases. Detailed data per gene are provided hereafter and illustrated in figure 1.

\section{Performances in BRAF analyses}

Three different types of Idylla cartridges were used for $B R A F$ analysis. ${ }^{916-18}$ 20-23 2526 The Idylla BRAF Mutation Test (CE-IVD approved) was used in seven studies whereas the Idylla NRASBRAF-EGFRS492R Mutation Assay (research use only) and the Idylla NRAS-BRAF Mutation Test (CE-IVD approved) were each used in one study. Overall, the rate of non-contributive results with these cartridges was $1.8 \%$ (25/1402). The concordance rates with the reference methods of the three cartridges were respectively $95.5 \%$ (1031/1079), 100\% (53/53) and 100\% (242/242); thus, discrepant results were only reported when using the Idylla BRAF cartridge $(4.4 \% ; 48 / 1079)$. Considering these cases, a BRAF mutation was detected solely by Idylla in $70.8 \%(34 / 48)$ of instances, whereas a BRAF mutation was detected by the reference method but not by Idylla in $22.9 \%$ $(11 / 48)$ of discrepant cases. Different BRAF mutations were instead detected by the two methods in $6.3 \%$ (3/48) of discrepant cases. Extremely rare BRAF mutations not included in the reference range (see table 2 for the list of mutations of the different Idylla cartridges) were responsible of Idylla-negative results in three cases. Sample cross-contamination, occurring during the preanalytical tissue handling, caused an Idylla false-positive result in one case. Third-line analyses allowed to confirm Idylla results in $58.3 \%(28 / 48)$ of discrepant results. In $31.3 \%$ $(15 / 48)$ of cases, no further explanation was given to explain the discrepancies.

\section{Performances in KRAS analyses}

The Idylla KRAS Mutation Test (CE-IVD) was used for KRAS analysis in seven studies with a rate of non-contributive results of $2.2 \%(18 / 808) .{ }^{10-12} 14181924$ The concordance of the Idylla test compared with the reference method (when this latter was contributive for the analysis of KRAS) was $92.1 \%$ (680/738). Among the 58 discrepant results, a KRAS mutation was detected only by Idylla but not by the comparison method in $55.2 \%(32 / 58)$ of cases, whereas the converse occurred in $37.9 \%(22 / 58)$ of cases. Different mutations were detected in $6.9 \%(4 / 58)$ of discrepant cases. Among discrepant cases, a third method suggested false-negative results of the first-line comparison methods in 32.8\% (19/58) of cases and of Idylla in $63.8 \%(37 / 58)$ of cases (no further analyses in 3.5\% (2/58) of cases). Reasons for Idylla false-negative results included a KRAS mutant allelic frequency $<5 \%$, below the threshold detectable by Idylla, rare mutations not included in the of Idylla KRAS Mutation Test reference range and technical problems related to malfunctioning cartridges. ${ }^{10}$ 


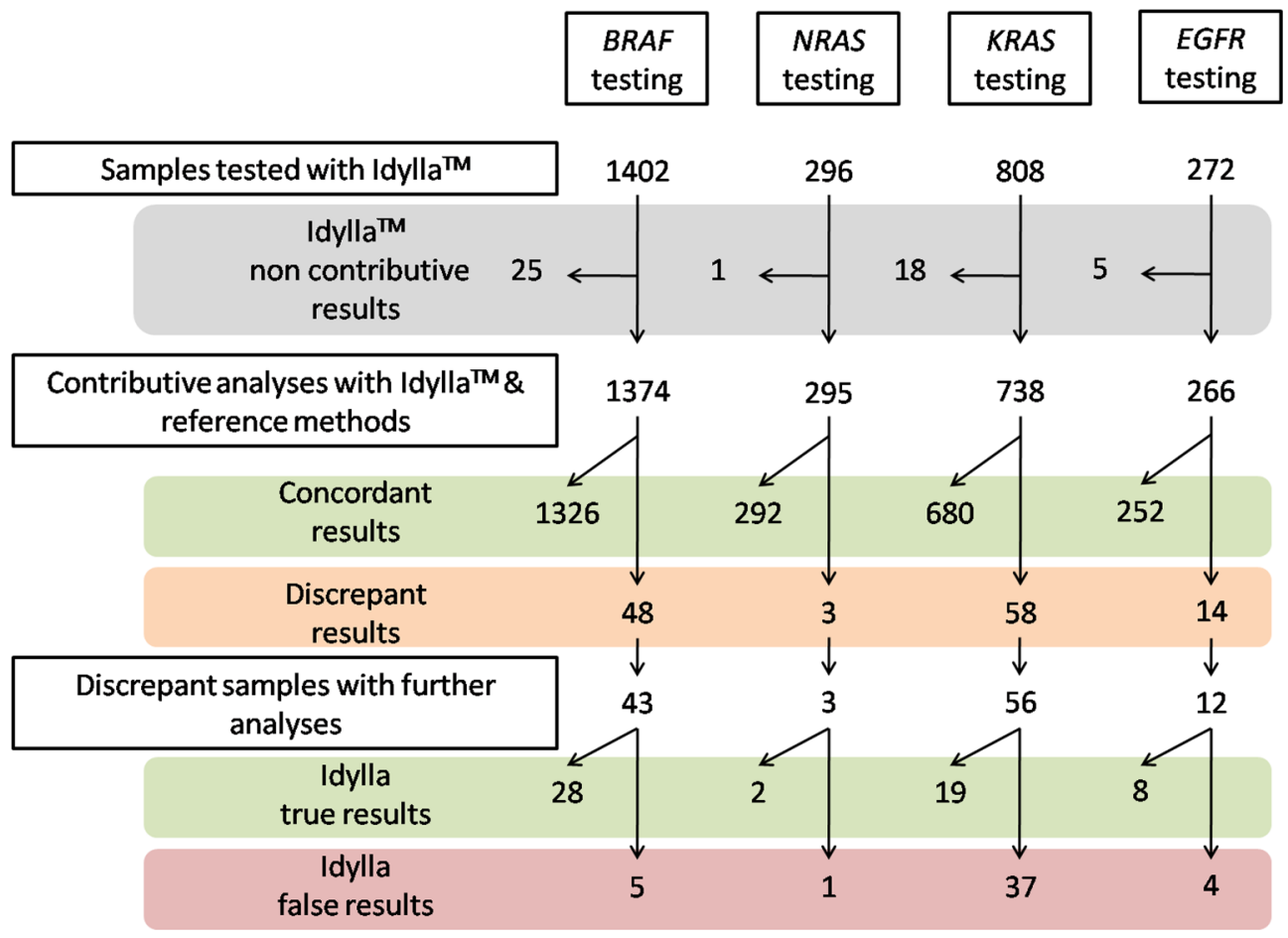

Figure 1 Graphical summary of the results using different Idylla tests compared with other molecular methods.

\section{Performances in NRAS analyses}

As mentioned above, the NRAS analyses were carried out by using two different Idylla cartridge types; in detail, the Idylla NRASBRAF-EGFRS492R Mutation Assay and the Idylla NRAS-BRAF Mutation Test cartridges were used in two and one study, respectively. ${ }^{17} 1826$ The use of a more recently released cartridge, designed for NRAS analysis alone (Idylla NRAS Mutation Test), has not been reported in the literature yet. Overall, out of a total of 296 tests performed, only the result of a single test was non-contributive. The concordance with the reference method was $98.9 \%(292 / 295)$ with only three discrepant results $(2 / 53$ cases with the Idylla NRAS-BRAF-EGFRS492R Mutation Assay and 1/242 cases with the Idylla NRAS-BRAF Mutation Test). A third method confirmed the Idylla results in two discordant cases harbouring low allelic frequencies NRAS mutations. In a third discordant case, additional molecular analyses showed a NRAS G13C mutation mutation. This was not included in the mutation covered by Idylla, whose result was a NRAS G12A/D mutation. ${ }^{26}$

\section{Performances in EGFR analyses}

Although the CE-IVD-approved Idylla EGFR Mutation Test has recently become available, literature data are based on four studies, carried out by the RUO Idylla EGFR Mutation Assay. ${ }^{101319}$ On a total of 272 tests performed with this cartridge type, only 5 tests $(1.8 \%)$ were non-contributive. Considering the data relative to the comparison of the 266 Idylla to the reference method, 14 (5.3\%) cases showing discrepancy in EGFR mutational status were reported. Thus, the concordance between Idylla and the first-line comparison method was $94.7 \%(252 / 266)$. In four cases, Idylla detected EGFR mutations that had been missed by the first-line comparison methods (fragment length and TaqMan assays) and, for two of these cases, a subsequent NGS analysis confirmed the mutations detected by Idylla. In one case, an Idylla software misinterpretation led to the erroneous detection of a EGFR exon 20 insertion besides two G719X and S768I mutations whereas only the G719X and S768I mutations were detected by NGS in this case. Idylla also detected a L861Q mutation in one case and T790M mutations in two cases, whereas pyrosequencing failed to detect these mutations (a third method confirmed Idylla results in these three cases). Nevertheless, in three samples, low allelic frequency EGFR T790M (NGS data) were not detected by Idylla.

\section{DISCUSSION}

The timely delivery of a biomarker testing result is a very crucial requirement to guide the therapeutic management of patients with advanced cancers, especially in case of acute deterioration. Beyond their expertise in tumour histopathology, as a matter of fact pathologists supervise the analytical workflow of immunohistochemical (IHC) and fluorescent in situ hybridisation assays, rendering accurate microscopic interpretation of relevant predictive expression, such as $A L K$, ROS1 and PD-L1. Conversely, molecular genetics mutational analyses often are not performed in surgical pathology laboratories but outsourced in reference molecular biology laboratories, which do not always hire a staff pathologist to take care of the crucial preanalytical steps related to microdissection for tumour cell enrichment. Ideally, novel technological advances could be instrumental for pathologists to perform molecular genetics analyses in-house at the time of the histopathological diagnosis, thus reducing testing turnaround time and allowing rapid treatment choices for the patients.

The Idylla device is a fully automated medical device able to perform molecular analyses in a few hours (between about 1 to 3 hours). To date, Idylla cartridges are available as CE-IVD-certified tools to analyse the most established actionable biomarkers. Indeed, using Idylla cartridges, it is feasible to test for EGFR and KRAS mutations in lung cancer samples, as well as KRAS, NRAS and BRAF mutations in colorectal carcinoma samples or $B R A F$ and NRAS mutations in melanoma samples with an excellent concordance with other classically used molecular methods. The implementation and use of Idylla does not require neither staff that has especially been trained in molecular analyses nor 
Table 2 Panels of the mutations detected the different Idylla cartridges

\begin{tabular}{|c|c|}
\hline Idylla BRAF Mutation Test (CE-IVD) & Idylla EGFR Mutation Test (CE-IVD) \\
\hline BRAF V600E (c.1799T>A; c.1799_1800delinsAA) & Exon 18 \\
\hline BRAF V600D (c.1799_1800delinsAT*; c.1799_1800delinsAC) & EGFR G719A (c.2156G>C) \\
\hline BRAF V600K (c.1798_ 1799delinsA A) & EGFR G719C (c.2155G>T;C.2154_2155delinsTT) \\
\hline BRAF V600R (c.1798_1799delinsAG) & EGFR G719S (c.2155G>A) \\
\hline BRAF V600M (c.1798G $>A)^{*}$ & Exon 19 \\
\hline Idylla NRAS Mutation Test (CE-IVD) & Del9 \\
\hline NRAS G12C (c.34 G>T) & (c.2238_2248delinsGC; c.2239_2248delinsC; c.2240_2248del; c.2239_2247del) \\
\hline NRAS G12S (c.34 G>A) & Del12 \\
\hline NRAS G12D (c.35G>A) & (c.2239_2251delinsC;c.2240_2251del) \\
\hline NRAS G12A (c.35G >C) & Del15 \\
\hline NRAS G12V (c.35G $>\mathrm{T})$ & (c.2235_2249del; c.2236_2250del; c.2239_2253del; c.2240_2254del; \\
\hline NRAS G13D (c.38G>A) & c.2238_2252del; c.2237_2251del; c.2235_2252delinsAAT; c.2237_2252delinsT; \\
\hline NRAS G13V (c.3 8G>T) & c.2234_2248del; c.2236_2253delinsCTA; c.2237_2253delinsTA; c.2237_2253delinsTC; \\
\hline NRAS G13R (c.37G >C) & c.2235_2251delinsAG; c.2236_2253delinsCAA; c.2230_2249delinsGTCAA) \\
\hline NRAS A59T (c.175G $>A)$ & Del18 \\
\hline NRAS Q61K (c.181C >A) & (c.2240_2257del; c.2237_2255delinsT; \\
\hline NRAS Q61L (c.182A>T) & c.2239_2256del; c.2236_2253del; \\
\hline NRAS Q61R (c.182A>G) & c.2239_2258delinsCA; c.2237_2254del; \\
\hline NRAS Q61H (c.183A>C; c.183A>T) & c.2238_2255del; c.2237_2257delinsTCT; \\
\hline NRAS K117N (c.351G>C; c.351G>T) & c.2236_2255delinsAT; c.2236_2256delinsATC; \\
\hline NRAS A146T (c.436G>A) & c.2237_2256delinsTT; c.2237_2256delinsTC; \\
\hline NRAS A146V (c.437C $>$ T) & c.2235_2255delinsGGT) \\
\hline Idylla NRAS-BRAF Mutation Test (CE-IVD) & Del21 \\
\hline Same panels as Idylla BRAF Mutation Test (CE-IVD) and Idylla NRAS Mutation Test (CE- & (c.2238_2258del; c.2236_2256del) \\
\hline $\begin{array}{l}\text { Same panels as Idylla BRAF Mutation Test (CE-IVD) and ldylla NRAS Mutation Test (CE- } \\
\text { IVD) except BRAF mutations marked with an * }\end{array}$ & $\begin{array}{l}\text { Del24 (c.2253_2276del) } \\
\text { Exon } 20\end{array}$ \\
\hline Idylla NRAS-BRAF-EGFR S492R Mutation Assay (RUO) & EGFR T790M (c.2369C>T) \\
\hline $\begin{array}{l}\text { Same panels as Idylla BRAF Mutation Test (CE-IVD) and Idylla NRAS Mutation Test (CE- } \\
\text { IVD) except BRAF mutations marked with an * . Detects also EGFR S492R (c.1476C }>A \text {; } \\
\text { c. } 1474 \mathrm{~A}>\text { C) mutations. }\end{array}$ & $\begin{array}{l}\text { EGFR S768I (c.2303G>T) insG (c.2310_2311insGGT) insASV9 } \\
\text { (c.2308_2309insGCCAGCGTG) insASV11 (c.2308_2311delinsCCAGCGTGGAT) insSVD } \\
\text { (c.2311_2312insGCGTGGACA) insH (c.2319_2320insCAC) }\end{array}$ \\
\hline Idylla KRAS Mutation Test (CE-IVD) & Exon 21 \\
\hline KRAS G12C (c.34G >T) & EGFR L858R (c.2573T>G;c.2573_2574delinsGT; \\
\hline $\begin{array}{l}\text { KRAS G12C (c.34G > I) } \\
\text { KRAS G12R (c.34G>C) }\end{array}$ & c.2573_2574delinsGA) \\
\hline \multirow{2}{*}{\multicolumn{2}{|c|}{ KRAS G12S (c.34G>A) }} \\
\hline & \\
\hline \multicolumn{2}{|l|}{ KRAS G12A (c.35G >C) } \\
\hline \multicolumn{2}{|l|}{ KRAS G12D (c.35G >A) } \\
\hline \multicolumn{2}{|l|}{ KRAS G12V (c.35G > T) } \\
\hline \multicolumn{2}{|l|}{ KRAS G13D (c.38G >A) } \\
\hline \multicolumn{2}{|l|}{ KRAS A59E (c.176C >A) } \\
\hline \multicolumn{2}{|l|}{ KRASA59G (c.176C>G) } \\
\hline \multicolumn{2}{|l|}{ KRAS A59T (c.175G>A) } \\
\hline \multicolumn{2}{|l|}{ KRAS Q61K (c.181C>A; c.180_181delinsAA) } \\
\hline \multicolumn{2}{|l|}{ KRAS Q61L (c.182 A>T) } \\
\hline \multicolumn{2}{|l|}{ KRAS Q61R (c.182A>G) } \\
\hline \multicolumn{2}{|l|}{ KRAS Q61H (c.183A>C; c.183A>T) } \\
\hline \multicolumn{2}{|l|}{ KRAS K117N (c.351A>C; c.351A>T) } \\
\hline \multicolumn{2}{|l|}{ KRAS A146P (c.436G>C) } \\
\hline \multicolumn{2}{|l|}{ KRAS A146T (c.436G >A) } \\
\hline KRAS A146V (c.437C>T) & \\
\hline
\end{tabular}

CE-IVD, European Community for in vitro diagnostic use.

dedicated laboratory rooms, representing a sustainable method for pathology laboratories, even without experience in molecular pathology.

Data generated by the studies listed in our review demonstrate high accuracy of the Idylla system to test for BRAF, NRAS, $K R A S$ and EGFR mutations in different cancers, underlining the cost-effectiveness of this approach with respect to other molecular methods. ${ }^{27}$ Local issues require local solution and depending on laboratory expertise and resources, Idylla can both represent a stand-alone diagnostic device or it can be included in a more complex diagnostic algorithm. The Idylla cartridges are designed to detect a wide range of mutations occurring in given oncogenes; thus the technique is highly accurate to detect more frequent mutations, whereas very rare and/or complex genomic variants, which are not included in the reference ranges, cannot be detected by the Idylla system. Hence, the occurrence of rare false-negative results should be taken into account as inevitable instances, when this system is used in a stand-alone manner. However, it is should be borne in mind that limitations do not apply to the Idylla system only as it is widely held that none of the molecular methods currently used in this field has perfect performances. The data reviewed in this study showed that in most of the discordant cases a third method confirmed the original assessment by Idylla. Being easy to use and rapid, the Idylla molecular method can be performed to reduce turnaround time as a first-line diagnostic tool, while a more comprehensive NGS analysis can be carried out a second time. Alternatively, Idylla can be used as an orthogonal technique to confirm, in a short time, uncertain results generated by the first-line use of a laboratory-developed technique (see figure 2). 


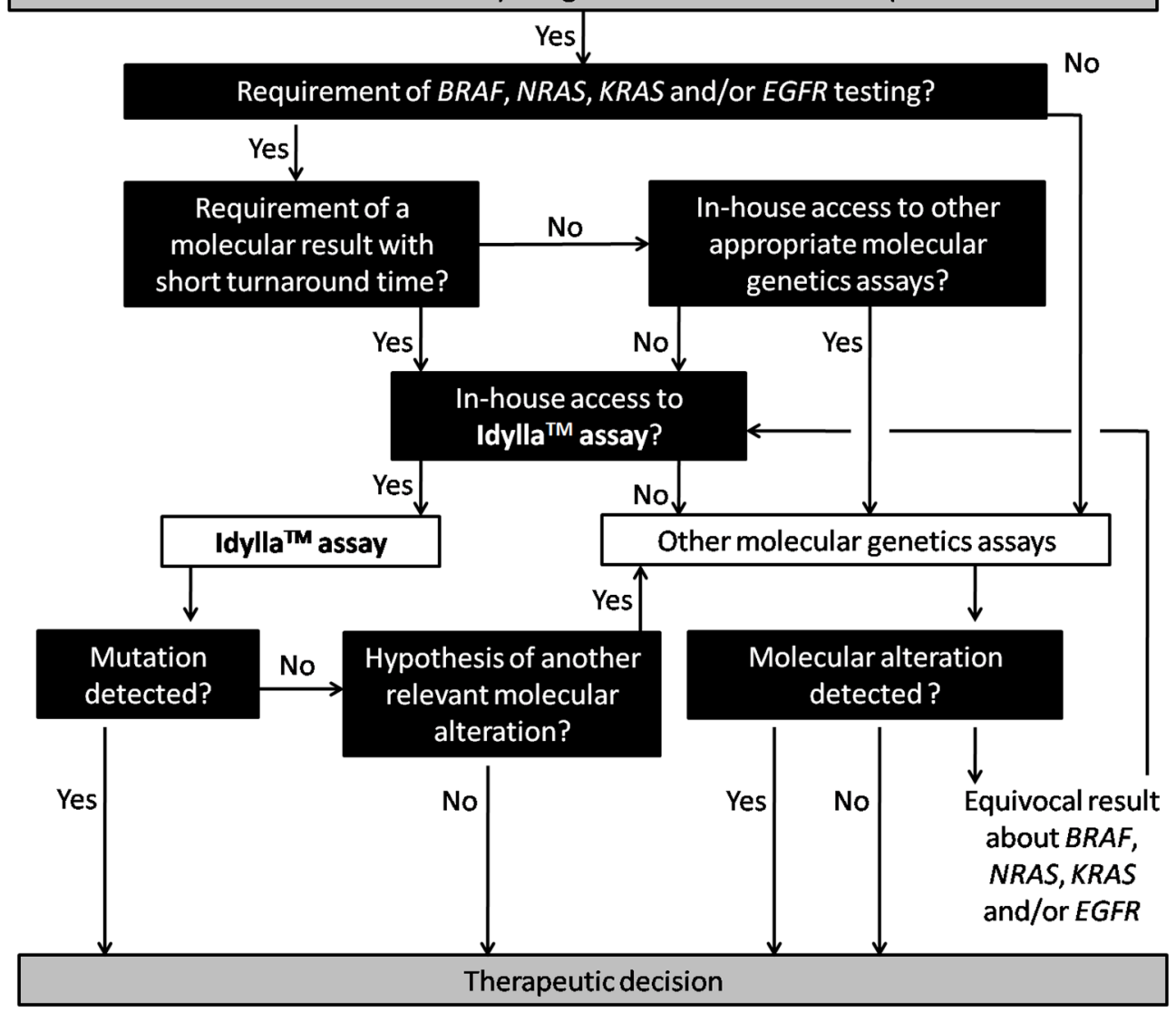

Figure 2 Proposal of a decision algorithm incorporating the Idylla assay with other molecular assays in laboratories practising molecular pathology.

With the progress of personalised medicine, searching for resistance mechanisms to explain and to anticipate cancer clinical relapse will become more and more important. Idylla can be useful to test for the EGFRT790M point mutation, the most frequent resistance mutations arising in patients with lung cancers treated with EGFR-dedicated tyrosine kinase inhibitors. However, data on the performance of Idylla in this setting are still limited and it can be argued that the subclonal and heterogeneous EGFRT790M tissue distribution may require a more sensitive approach. As a general rule, we believe that the wide variety of the potential molecular mechanisms potentially implicated in resistance makes Idylla analyses out of the field, to date. In this setting, the analysis of large gene panels may be more effective to cover the various molecular mechanisms underlying resistance to therapies, to maximise the identification of a molecular target and clinical trial access. In this manner, we strongly believe that Idylla's role in the complex genomic predictive biomarker testing landscape mostly consists of standard-of-care first-line diagnostic analyses for the rapid and initial management of patients with cancer. As it does not require large amounts of tumour tissue (eg, a single $10 \mu \mathrm{m}$ tissue section of a lung biopsy is sufficient for Idylla EGFR Mutation Test), first-line Idylla testing for rapid initial treatment choices does not prevent in the meantime the initiation of additional more extensive genotyping to provide additional important information for the future management of patients. Nevertheless, Idylla could also be a valuable tool for the monitoring of patients with cancers because, beyond the scope of this review, other cartridges dedicated to cell-free circulating tumour DNA analyses in liquid biopsies are also developed with the same easy-to-use and fast-turnaround time features as those dedicated to pathology samples reviewed herein. ${ }^{28} 29$

To conclude, the Idylla system is a valuable, fast and easyto-use diagnostic platform for molecular genotyping. In addition to the currently available tests dedicated to $B R A F, N R A S$, $K R A S$ and EGFR genotyping, new cartridges are being developed. The Idylla system testing for new biomarkers required for the therapeutic managements of patients (eg, microsatellite instability) will increase the capacity of pathologists to deliver quickly molecular information relevant to immediate treatment choices at the time of diagnosis. ${ }^{30}$ This first-line quick molecular diagnosis will not impair further more extensive but also more time-consuming genotyping for subsequent patients' management. Coupling with morphological IHC and in situ hybridisation analyses, pathologists have now with Idylla the opportunity to deliver a molecular diagnosis in one single day for the early management of patients with advanced cancers who are candidates for targeted therapies.

\section{Take home messages}

We reviewed 18 studies evaluating the Idylla diagnostic platform.

- BRAF, KRAS, NRAS and EGFR analyses were considered.

- The concordance between Idylla and reference methods was excellent.

- Idylla permits a fast molecular diagnosis in 1 day for patients with advanced cancers. 
Handling editor Runjan Chetty.

Contributors Both AU and GT collected the data, drafted the manuscript and approved its final version.

Funding The authors have not declared a specific grant for this research from any funding agency in the public, commercial or not-for-profit sectors.

Competing interests None declared.

Patient consent Not required.

Provenance and peer review Not commissioned; externally peer reviewed.

(c) Article author(s) (or their employer(s) unless otherwise stated in the text of the article) 2018. All rights reserved. No commercial use is permitted unless otherwise expressly granted.

\section{REFERENCES}

1 Benson AB, Venook AP, Cederquist L, et al. Colon Cancer, version 1.2017, NCCN clinical practice guidelines in oncology. J Nat/ Compr Canc Netw 2017;15:370-98.

2 Lindeman NI, Cagle PT, Aisner DL, et al. Updated molecular testing guideline for the selection of lung cancer patients for treatment with targeted tyrosine kinase inhibitors: guideline from the college of american pathologists, the international association for the study of lung cancer, and the association for molecular pathology. Arch Pathol Lab Med 2018;142:321-46.

3 Gradishar WJ, Anderson BO, Balassanian R, et al. NCCN guidelines insights breast cancer, version 1.2016. J Nat/ Compr Canc Netw 2015;13:1475-85.

4 Ajani JA, D'Amico TA, Almhanna K, et al. Gastric cancer, version 3.2016, NCCN clinical practice guidelines in oncology. J Natl Compr Canc Netw 2016;14:1286-312.

5 Coit DG, Thompson JA, Algazi A, et al. NCCN guidelines insights: melanoma, version 3.2016. J Nat/ Compr Canc Netw 2016;14:945-58.

6 Capoluongo E, Ellison G, López-Guerrero JA, et al. Guidance statement on BRCA1/2 tumor testing in ovarian cancer patients. Semin Oncol 2017;44:187-97.

7 von Mehren M, Randall RL, Benjamin RS, et al. Gastrointestinal stromal tumors, version 2.2014. J Nat/ Compr Canc Netw 2014;12:853-62.

8 Guo Z, Lloyd RV. Use of monoclonal antibodies to detect specific mutations in formalin-fixed, paraffin-embedded tissue sections. Hum Pathol 2016;53:168-77.

9 Melchior L, Grauslund M, Bellosillo B, et al. Multi-center evaluation of the novel fully-automated PCR-based IdyllaTM BRAF Mutation Test on formalin-fixed paraffinembedded tissue of malignant melanoma. Exp Mol Pathol 2015;99:485-91.

10 Thomas De Montpréville V, Ghigna MR, Lacroix L, et al. EGFR and KRAS molecular genotyping for pulmonary carcinomas: Feasibility of a simple and rapid technique implementable in any department of pathology. Pathol Res Pract 2017;213:793-8.

11 Weyn C, Van Raemdonck S, Dendooven R, et al. Clinical performance evaluation of a sensitive, rapid low-throughput test for KRAS mutation analysis using formalin-fixed, paraffin-embedded tissue samples. BMC Cancer 2017;17:139.

12 De Luca C, Vigliar E, d'Anna M, et al. KRAS detection on archival cytological smears by the novel fully automated polymerase chain reaction-based Idylla mutation test. Cytojournal 2017;14:5.

13 De Luca C, Gragnano G, Pisapia P, et al. EGFR mutation detection on lung cancer cytological specimens by the novel fully automated PCR-based Idylla EGFR Mutation Assay. J Clin Pathol 2017;70:295-300.

14 de Biase D, de Luca C, Gragnano G, et al. Fully automated PCR detection of KRAS mutations on pancreatic endoscopic ultrasound fine-needle aspirates. J Clin Pathol 2016.
15 Ilie M, Butori C, Lassalle $S$, et al. Optimization of EGFR mutation detection by the fullyautomated qPCR-based Idylla system on tumor tissue from patients with non-small cell lung cancer. Oncotarget 2017;8:103055-62.

16 Bisschop C, Ter Elst A, Bosman LJ, et al. Rapid BRAF mutation tests in patients with advanced melanoma: comparison of immunohistochemistry, Droplet Digital PCR, and the Idylla Mutation Platform. Melanoma Res 2018;28:96-104.

17 Johnston L, Power M, Sloan P, et al. Clinical performance evaluation of the Idylla NRAS-BRAF mutation test on retrospectively collected formalin-fixed paraffinembedded colorectal cancer tissue. J Clin Pathol 2018;71:336-43.

18 Colling R, Wang LM, Soilleux E. Validating a fully automated real-time PCR-based system for use in the molecular diagnostic analysis of colorectal carcinoma: a comparison with NGS and IHC. J Clin Pathol 2017;70:610-4.

19 Lambros L, Caumont C, Guibourg B, et al. Evaluation of a fast and fully automated platform to diagnose EGFR and KRAS mutations in formalin-fixed and paraffinembedded non-small cell lung cancer samples in less than one day. J Clin Pathol 2017;70:544-9.

20 Yeo MK, Jung MK, Lee SY, et al. The usefulness of a novel fully automated PCRbased Idylla test for detection of the BRAF V600E mutation in thyroid tissue: comparison with PNA-clamping PCR, real-time PCR and pyrosequencing. J Clin Pathol 2017;70:260-5.

21 Schiefer Al, Parlow L, Gabler L, et al. Multicenter evaluation of a novel automated rapid detection system of BRAF status in formalin-fixed, paraffin-embedded tissues. J Mol Diagn 2016;18:370-7.

22 Colling R, Wang LM, Soilleux E. Automated PCR detection of BRAF mutations in colorectal adenocarcinoma: a diagnostic test accuracy study. J Clin Pathol 2016;69:398-402.

23 Janku F, Claes B, Huang HJ, et al. BRAF mutation testing with a rapid, fully integrated molecular diagnostics system. Oncotarget 2015;6:26886-94.

24 Solassol J, Vendrell J, Märkl B, et al. Multi-Center evaluation of the fully automated PCR-based IdyllaTM KRAS mutation assay for rapid KRAS mutation status determination on formalin-fixed paraffin-embedded tissue of human colorectal cancer. PLoS One 2016;11:e0163444.

25 Harlé A, Salleron J, Franczak C, et al. Detection of BRAF mutations using a fully automated platform and comparison with high resolution melting, real-time allele specific amplification, immunohistochemistry and next generation sequencing assays, for patients with metastatic melanoma. PLoS One 2016;11:e153576.

26 Barel F, Guibourg B, Lambros L, et al. Evaluation of a rapid, fully automated platform for detection of BRAF and NRAS mutations in melanoma. Acta Derm Venereol 2018;98:44-9.

27 Le Flahec G, Guibourg B, Marcorelles P, et al. Financial implications of Idylla testing in colorectal cancer, lung cancer and melanoma: a French laboratory point of view. J Clin Pathol 2017;70:906-7.

28 Janku F, Huang HJ, Claes B, et al. BRAF mutation testing in cell-free DNA from the plasma of patients with advanced cancers using a rapid, automated molecular diagnostics system. Mol Cancer Ther 2016;15:1397-404.

29 Seremet T, Planken S, Schreuer M, et al. Illustrative cases for monitoring by quantitative analysis of BRAF/NRAS ctDNA mutations in liquid biopsies of metastatic melanoma patients who gained clinical benefits from anti-PD1 antibody therapy. Melanoma Res 2018;28:65-70.

30 Maertens G, De Craene B, Rondelez E, et al. 138PDetection of microsatellite instability (MSI) in colorectal cancer samples with the automated Idylla MSI Test. Annals of Oncology 2017;28(suppl_5):138P. 